social distancing measures introduced during the COVID-19 pandemic. We identified the need to seek feedback from the patients attending these clinics.

Methods We designed a questionnaire for patients who had attended a virtual clinic in General Surgery, using both Qualitative and Quantitative questions. We contacted 100 patients who attended a range of clinics ran by over 10 different General Surgical Consultants between 25th March 2020 and 30th April 2020. A team of junior doctors and clinical assistants were recruited to contact patients. Patients were contacted via telephone to ask if they would be happy to complete the survey which would be emailed to them, if they did not have an email address a shorter survey was completed over the phone.

Results In total we had 73 responses to the survey. Data collected suggests that virtual clinics are well received by patients. $48 \%(n=35)$ answered that they were overall 'very satisfied' with the care received in the virtual clinic, and $79.6 \%(n=43)$ felt that their care did not suffer as a result of non-face to face contact. Most patients would recommend a virtual clinic to their friend or family $77 \%(n=56)$, however if given the option $60 \% \quad(n=43)$ would prefer a face to face clinic appointment.

Conclusions As the pandemic progresses it is important to identify ways in which good patient care can be delivered whilst not putting patients at risk of contracting the virus. Virtual clinics not only maintain patient safety but also have many other benefits to patients as identified in this study. This study suggests that perhaps the use of virtual clinics should continue and could be utilised by surgical departments to provide efficient patient centred care.

\section{Developing effective leaders}

\section{ENHANCING JUNIOR DOCTOR LEADERSHIP AND MANAGEMENT SKILLS AS INNOVATION FELLOWS}

Orod Osanlou, Kate Clark, Bethany Griffiths, Michaela Morton, Muni Matoug*, Ricia Gwenter, Richard Brown, Hafsa Khan, Carys Phillips. Wrexham Maelor Hospital, Betsi Cadwaladr University Health Board

\subsection{6/leader-2020-FMLM. 188}

Aims The Site Innovation Lead at Wrexham Maelor Hospital, Betsi Cadwaladr University Health Board appointed 12 junior doctors as Innovation Fellows in order to enhance their leadership and management skills.

Methods Junior doctors competitively applied to be a Wrexham Innovation Fellow. This formal leadership position included mentoring from the Site Innovation Lead and quality improvement (QI) training. Each Innovation Fellow was guided and encouraged to undertake the Improving Quality Together' (IQT) Welsh QI Development Programme as well as the Edward Jenner NHS Leadership Academy Programme. Funding has also been secured to deliver further formal QI training from Improvement Cymru. These skills have then been used by the Innovation Fellows when undertaking QI and innovation work.

Results As of August 2020, 8/12 (75\%) of Innovation fellows completed the Improving Quality Together Welsh QI Development Programme and 5/11 (45\%) have completed the Edward Jenner NHS Leadership Academy Programme. 10/12 (83\%) feel that their leadership skills have improved since becoming an Innovation Fellow. 11/12 (92\%) feel more motivated to undertake QI and innovation since becoming an Innovation Fellow, and 10/12 (83\%) feel more motivated to support others undertake QI and innovation since starting the programme. 10/11 (91\%) also feel that their leadership experience may better equip them in the planning and management of future COVID challenges.

Conclusions Empowering junior doctors with leadership responsibilities while also providing mentorship and formal QI training can enhance their leadership skills, motivate them to undertake QI and innovation, and empower them to supervise and mentor others. Other NHS organisations should consider appointing Innovation Fellows in a similar way to Betsi Cadwaladr University Health Board.

\section{Lead sepsis improvement nurse}

\section{EVALUATION OF A STRUCTURED TOOL FOR ESCALATING DETERIORATING PATIENTS FOR MULTIDISCIPLINARY STAFF IN A LARGE LONDON TEACHING HOSPITAL?}

Daminee Seetannah, Dalia Ludwig. Daminee Seetannah, University College London Hospital, UK

\subsection{6/leader-2020-FMLM.189}

The lead sepsis nurse, chief registrar and simulation team in a large London teaching hospital have developed a digital communication tool to improve and standardise the process of escalation and handover of deteriorating patients. The tool is designed for use by the multidisciplinary team (MDT). Poor communication is a frequently cited causative factor leading to adverse clinical events, particularly in relation to escalation and handover of care. UCLH uses ISBARD (Introduction, situation, background, assessment, recommendation and decision) to facilitate and improve how we escalate and handover deteriorating patients. Our trust switched to an electronic health record system last year using the software Epic. Since the introduction of Epic, we have had two serious incidents (SIs) related to miscommunication of escalation of NEWS2 scores. Investigation of these SIs concluded that the process of escalating a rising NEWS2 score could be improved by the use of an ISBARD tool creating a common language to escalate a deteriorating patient. Therefore, we have built a quick and intuitive ISBARD smart text in Epic to use when escalating a deteriorating patient. Teaching sessions are booked for the MDT over the next few months to train staff in the environment where they will use the tool to illustrate its use. We are working with the simulation team to embed the tool in clinical SIM training. We involved the MDT in design and implementation from the outset to optimise staff engagement. If we were starting again we would involve leaders of different staff groups at an earlier stage to help promote the importance and value of the tool. Our project is to streamline staff workload and help identify high risk wards where additional support may be needed. Our tool is designed to aid communication and empower clinicians to escalate their concerns safely and prioritise patient care. 